\title{
Efficacy of Endocuff Vision compared to first-generation Endocuff in adenoma detection rate and polyp detection rate in high-definition colonoscopy: a systematic review and network meta-analysis
}

\section{다(1)우우}

\author{
Authors \\ Muhammad Aziz* 1, Hossein Haghbin*2, Manesh Kumar Gangwani³, Sachit Sharma ${ }^{4}$, Yusuf Nawras², Zubair Khan ${ }^{5}$, \\ Saurabh Chandan ${ }^{6}$, Babu P. Mohan ${ }^{7}$, Wade Lee-Smith ${ }^{8}$, Ali Nawras ${ }^{1}$
}

Institutions

1 Division of Gastroenterology and Hepatology, University of Toledo Medical Center, Toledo, Ohio, United States

2 Department of Internal Medicine, University of Toledo Medical Center, Toledo, Ohio, United States

3 Department of Internal Medicine, Mercy Hospital St. Louis, St. louis, Missouri, 63141

4 Department of Internal Medicine, University of Toledo and Promedica Toledo Hospital, Toledo, Ohio, United States

5 Department of Gastroenterology, McGovern Medical School, University of Texas Health Science Center at Houston, Houston, Texas, United States

6 Division of Gastroenterology and Hepatology, $\mathrm{CHI}$ Creighton University Medical Center, Omaha, Nebraska, United States

7 Divison of Gastroenterology and Hepatology, University of Utah Healthcare, Salt Lake City, Utah, United States

8 University of Toledo Libraries, University of Toledo Medical Center, Toledo, Ohio, United States

submitted 16.7.2020

accepted after revision $\quad 30.9 .2020$

Bibliography

Endoscopy International Open 2021; 09: E41-E50

DOI 10.1055/a-1293-7327

ISSN 2364-3722

(C) 2021. The Author(s).

This is an open access article published by Thieme under the terms of the Creative Commons Attribution-NonDerivative-NonCommercial License, permitting copying and reproduction so long as the original work is given appropriate credit. Contents may not be used for commecial purposes, or adapted, remixed, transformed or built upon. (https://creativecommons.org/licenses/by-nc-nd/4.0/)

Corresponding author

Muhammad Aziz, MD, University of Toledo Medical Center, 3000 Arlington Avenue, Toledo, Ohio, 43614, United States Fax: +1-816-204-0605

marajani@hotmail.com
\# Supplementary material is available under https://doi.org/10.1055/a-1293-7327

\section{ABSTRACT}

Background and study aims Recently, the newer Endocuff Vision (ECV) has been evaluated for improving colonoscopy outcome metrics such as adenoma detection rate (ADR) and polyp detection rate (PDR). Due to lack of direct comparative studies between ECV and original Endocuff (ECU), we performed a systematic review and network meta-analysis to evaluate these outcomes.

Methods The following databases were searched: PubMed, Embase, Cochrane, and Web of Sciences to include randomized controlled trials (RCTs) comparing ECV or ECU colonoscopy to high-definition (HD) colonoscopy. Direct as well as network meta-analyses comparing ADR and PDR were performed using a random effects model. Relative-risk (RR) with $95 \%$ confidence interval $(\mathrm{Cl})$ was calculated.

Results A total of 12 RCTs with 8638 patients were included in the final analysis. On direct meta-analysis, ECV did not demonstrate statistically improved ADR compared to HD colonoscopy (RR: 1.12, $95 \% \mathrm{Cl} 0.99-1.27$ ). A clinically and statistically improved PDR was noted for ECV compared to HD (RR: $1.15,95 \% \mathrm{Cl} 1.03-1.28$ ) and ECU compared to HD (RR: $1.26,95 \% \mathrm{Cl} 1.09-1.46$ ) as well as improved ADR (RR: $1.22,95 \% \mathrm{Cl} 1.05-1.43$ ) was observed for ECU colonoscopy when compared to HD colonoscopy. These results were also consistent on network meta-analysis. Lower overall complication rates (RR: $0.14,95 \% \mathrm{Cl} 0.02-0.84$ ) and particularly lacerations/erosions (RR: $0.11,95 \% \mathrm{Cl} 0.02-0.70$ ) were noted with ECV compared to ECU colonoscopy.

Conclusions Although safe, the newer ECV did not significantly improve ADR compared to ECU and HD colonoscopy. Further device modification is needed to increase the overall ADR and PDR.

* These authors contributed equally. 


\section{Introduction}

Colorectal cancer (CRC) is the second and fourth leading cause of cancer death in the United States and around the world, respectively [1]. The estimated deaths from CRC in 2019 were 51,020 in the United States, according to the SEER database [2]. The majority of the CRCs ( 80\%) develop through the well-studied adenoma-carcinoma sequence while a subset of CRCs ( 20\%) develop through the serrated adenoma pathway [3]. High-definition (HD) colonoscopy identifies adenomas and serrated lesions at early stage and reduces cancer-related morbidity and mortality [4]. Adenoma detection rate (ADR) - defined as proportion of colonoscopies with at least one adenoma detected - is a well-established colonoscopy indicator [4,5]. An improvement in ADR is associated with a significant reduction in interval CRC and mortality [5]. The ADR $\geq 30 \%$ for men and $\geq 20 \%$ for women is recommended for average risk screening colonoscopies [6].

Current efforts are directed to increase the ADR for endoscopists by using numerous interventions (electronic chromoendoscopy, add-on devices, antispasmodic medications, multiple observers, water-aided methods, second forward exam or retroflexion in right colon, artificial intelligence) [7-13]. Two such add-on devices used in recent times are Endocuff (ECU) and Endocuff Vision (ECV) (Arc Medical Design, Leeds, UK) [14]. The first-generation ECU included two rows of soft finger-like projections in order to flatten the colonic haustral folds while the newer second generation ECV includes only one row of finger-like projections with blunter tips to decrease the incidence of mucosal laceration and erosions [14, 15].

Recent meta-analyses in literature have compared the Endocuff devices to HD colonoscopy and a significantly improved ADR was noted for Endocuff colonoscopy. The major limitation of these meta-analyses is that both ECU and ECV were pooled together for generating outcomes [7, 16-19]. Further, data regarding the comparative efficacy of the two cuffs (i.e. ECU vs ECV) is not available in literature. We conducted an updated systematic review and network meta-analysis of all the available randomized controlled trials (RCTs) to generate the best possible evidence comparing the first- and second-generation Endocuff devices to each other and to HD colonoscopy.

\section{Methods}

A detailed and comprehensive search of the following databases were conducted from inception through March 25, 2020: PubMed/Medline, Embase, Web of Science Core Collection, and Cochrane Central Register of Controlled Trials. The keywords and search terms for the concepts of "colonoscopy", "adenoma/polyps", “detection rates”, and "Endocuff” were developed for EMBASE and translated to vocabulary for other databases. The search strategy was created by an experienced librarian (W.L.S.) and reviewed by another investigator (M.A.). Detailed search strategy for EMBASE is provided in Supplementary Table 1. Screening of the articles was performed by two independent reviewer (H.H. and M.A.) and discrepancy was resolved through mutual discussion. Preliminary screening was done using title and abstracts and full texts of relevant articles were obtained. Bibliographies of the included articles were also checked to see any additional articles fulfilled our study criteria. We adhered to Preferred Reporting Items for Systematic Reviews and Meta-Analyses (PRISMA) guidelines and no restriction to language was applied.

\section{Study definitions}

ADR is defined as the proportion of colonoscopies with at least one adenoma detected in all colonoscopies. Polyp detection rate (PDR) is defined as the proportion of colonoscopies with at least one polyp detected in all colonoscopies. The serrated adenoma detection rate (SADR) is defined as the proportion of colonoscopies with at least one sessile serrated adenoma/polyp (SSA/P) and/or traditional serrated adenoma (TSA) detected in all colonoscopies. The advanced adenoma detection rate (AADR) is defined as proportion of colonoscopies with at least one advanced adenoma (adenomas $\geq 10 \mathrm{~mm}$ or with villous components or high-grade dysplasia) in all colonoscopies. The neoplasia detection rate (NDR) is defined as proportion of colonoscopies with at least one neoplasia (intramucosal carcinoma and/or adenocarcinoma) in all colonoscopies. The mean adenoma per procedure (MAP) is defined as the total number of adenomas divided by the total number of procedures. The mean polyp per procedure (MPP) is defined as the total number of polyps divided by the total number of procedures. The cecal intubation rate (CIR) is defined as achieving cecal intubation during the insertion phase of colonoscopy.

\section{Inclusion/exclusion criteria}

We included randomized controlled trials (RCT) that met the following criteria: (A) Population: Adult patients (>18 years) undergoing colonoscopy (B) Intervention: First-generation ECU, and/or second-generation ECV utilization for colonoscopy. (C) Comparison: High-definition (HD) colonoscopy. Studies with colonoscopy performed using older standard definition colonoscopy were excluded. Studies comparing ECU or ECV with other devices (Endocap, Endoring, etc.) were also excluded (D) Outcomes: ADR and PDR. Studies were excluded if patients with obvious underlying pathology were included (inflammatory bowel disease, serrated polyposis syndrome, hyperplastic polyposis syndrome). Other types of studies (single arm, cohort, editorials, case series/reports, review articles) were also excluded. Abstracts were also excluded for the purpose of the manuscript.

\section{Data extraction and study outcomes}

Data from finalized studies including baseline demographics (age, gender, indication for colonoscopy), total study participants, type of intervention, study completion rate, quality indicators (cecal intubation time, withdrawal time), and study outcomes (PDR, ADR, AADR, NDR, SADR, MAP, and MPP) was collected and tabulated on Microsoft Excel (Microsoft, Redmond, Washington, United States) by two independent reviewers (H.H. and M.A.). Any discrepancy in data collection was resolved through mutual discussion. We used the per-protocol 
(PP) analysis with detection rates calculated based on actual study completion.

\section{Data synthesis and statistical analysis}

We performed direct meta-analysis to generate direct evidence for studies doing a head-to-head comparison between intervention groups. We also performed a network meta-analysis to generate direct and indirect evidence for the same. DerSimonian-Laird method and random effects model was used to perform a direct meta-analysis on 'Comprehensive Meta-Analysis' software (Biostat, Englewood, New Jersey, United States). Network meta-analysis was conducted using a random-effects model on 'R' package 'Netmeta' (Bell labs, Murray Hill, New Jersey, United States). Relative risk (RR) with $95 \%$ confidence interval $(\mathrm{Cl})$ for each proportional outcome was calculated. Mean difference (MD) with $95 \% \mathrm{Cl}$ was calculated for continuous variables. $P<0.05$ was considered statistically significant. The "frequentist method" was used to rank the intervention and a P score was generated [20]. A higher P score (closer to 1.00 ) correspond to superior detection rates for respective intervention group. For complications, a higher $\mathrm{P}$ score (closer to $1.00)$ correspond to lower complication rates for respective intervention. Study heterogeneity was assessed using the $\mathrm{I}^{2}$ statistic defined by the Cochrane Handbook for systematic reviews and value $>50 \%$ was considered as substantial heterogeneity [21]. Disagreement between direct and indirect evidence was assessed using the node splitting technique [22]. The Grading of Recommendations Assessment, Development and Evaluation (GRADE) approach was utilized for assessing the certainty of evidence and rating of very low, low, moderate and high was determined [23].

\section{Subgroup analysis}

Subgroup analysis was performed based on the ADR for control groups i.e. studies with control $A D R \leq 40 \%$ vs $A D R>40 \%$ and studies with control ADR $\leq 30 \%$ vs ADR $>30 \%$.

\section{Bias assessment}

The bias assessment for included RCTs was evaluated using the methodology outlined in Cochrane handbook for systematic reviews of interventions [24]. Publication bias was visually assessed using funnel plots as well as quantitatively assessed using Egger's regression analysis. $P<0.05$ was indicative of substantial publication bias.

\section{Results}

A total of 168 articles were screened after removing duplicates and using the search strategy mentioned above. Twelve RCTS were included after rigorous screening of abstracts and full texts with a total of 8638 patients (4285 in HD group, 4353 in Endocuff group) ( Fig. 1) [15,25-35]. All studies were published after 2014 . Study completion rate was $98.4 \%$ and $97.1 \%$ respectively for HD and Endocuff group.
327 records identified through database

searching

105 in Cochrane Library

108 in Embase

37 in Pubmed/Medline

77 in Web of Science

119 in Google Scholar

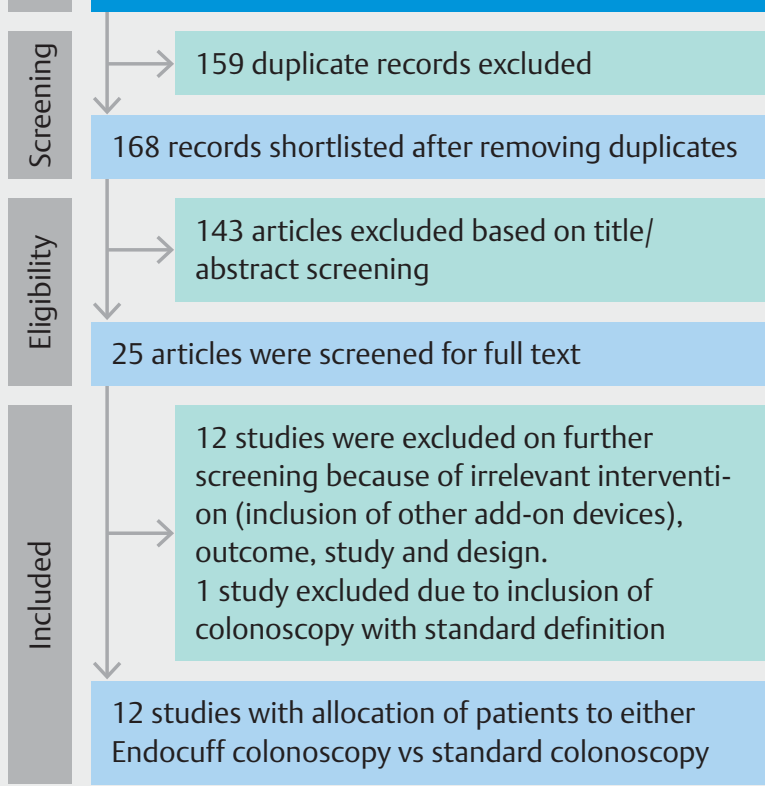

- Fig. 1 PRISMA flow diagram. From: Moher D, Liberati A, Tetzlaff J, Altman DG, The PRISMA Group (2009). Preferred Reporting Items for Systematic Reviews and Meta-Analyses: The PRISMA Statement. PLoS Med 6(7): e1000097. doi:10.1371/journal.pmed1000097

\section{Baseline demographics/study outcomes}

The groups were similar in terms of age range (55.7-68 vs $55.1-68)$, male gender (52.9\% vs $52.1 \%$ ) and screening/surveillance indication (60.8\% vs $60.7 \%$ ). Of the Endocuff group, 2627 patients (6 studies) underwent ECV colonoscopy[14, 25, $29,30,33,35$ ] and 1726 patients (6 studies) underwent ECU colonoscopy [26-28, 31, 32, 34] ( Table 1). The outcomes for individual studies are summarized in $>$ Table 2 .

\section{Direct meta-analysis}

A total of six studies assessed ADR and a statistically insignificant but improved detection trend was observed for ECV compared to HD colonoscopy $(42.6 \%$ vs $36.8 \%$, RR: $1.12,95 \% \mathrm{Cl}$ 0.99-1.27, $P=0.07, I^{2}=66.6 \%$ ) ( Fig. 2a) $[15,25,29,30,33]$. Six studies compared ECU to HD colonoscopy and improved ADR was observed for ECU colonoscopy that was statistically significant (48.3\% vs $41.1 \%$, RR: $1.22,95 \% \mathrm{Cl} 1.05-1.43, \mathrm{p}=$ $0.01, \mathrm{I}^{2}=71.4 \%$ ) ( Fig. 2b) $[26,27,28,31,32,34]$. A statistically and clinically significant improvement in PDR was noted for ECV colonoscopy compared to HD colonoscopy $(52.5 \%$ vs $45.5 \%$, RR: $1.15,95 \% \mathrm{Cl} 1.03-1.28, P=0.01, \mathrm{I}^{2}=67.0 \%$ ) (-Fig. 2c) $[15,25,29,33]$. Similarly, PDR was found to be improved for 
- Table 1 Baseline study characteristics and patient demographics.

\begin{tabular}{|c|c|c|c|c|c|c|c|c|}
\hline \multirow[t]{2}{*}{ Study, year } & \multirow{2}{*}{$\begin{array}{l}\text { Tech- } \\
\text { niques } \\
\text { compared }\end{array}$} & \multirow{2}{*}{$\begin{array}{l}\text { Total } \\
\text { Patients, } \\
\text { N }\end{array}$} & \multirow{2}{*}{$\begin{array}{l}\text { Study } \\
\text { comple- } \\
\text { tion, N }\end{array}$} & \multirow{2}{*}{$\begin{array}{l}\text { Mean/ } \\
\text { Median } \\
\text { Age, n }\end{array}$} & \multirow{2}{*}{$\begin{array}{l}\text { Male } \\
\text { propor- } \\
\text { tion, \% }\end{array}$} & \multicolumn{2}{|c|}{$\begin{array}{l}\text { Indication for } \\
\text { colonoscopy, \% }\end{array}$} & \multirow[t]{2}{*}{$\begin{array}{l}\text { Type of Colonoscope } \\
\text { used }\end{array}$} \\
\hline & & & & & & $\mathrm{HD}$ & ECU/ECV & \\
\hline $\begin{array}{l}\text { Biecker, } 2015 \\
{[26]}\end{array}$ & HD ECU & $\begin{array}{l}253 \\
245\end{array}$ & $\begin{array}{l}249 \\
240\end{array}$ & $\begin{array}{l}68 \\
65\end{array}$ & $\begin{array}{l}51.8 \% \\
47.8 \%\end{array}$ & $\begin{array}{l}\text { S: NR } \\
\text { D: NR }\end{array}$ & $\begin{array}{l}\text { S: NR } \\
\text { D: NR }\end{array}$ & $\begin{array}{l}\text { Fujifilm EC-590 WM4, } \\
\text { EC-590 WL4, Olympus } \\
\text { CF-H180 AI }\end{array}$ \\
\hline $\begin{array}{l}\text { De Palma, } 2018 \\
\text { [27] }\end{array}$ & HD ECU & $\begin{array}{l}144 \\
144\end{array}$ & $\begin{array}{l}137 \\
137\end{array}$ & $\begin{array}{l}55.7 \\
55.1\end{array}$ & $\begin{array}{l}52.6 \% \\
51.8 \%\end{array}$ & $\begin{array}{l}\text { S: } 66.4 \% \\
\text { D: } 33.6 \%\end{array}$ & $\begin{array}{l}\text { S: } 65.0 \% \\
\text { D: } 35.0 \%\end{array}$ & $\begin{array}{l}\text { Olympus CF-HQ190, } \\
\text { CF-Q180AL/I }\end{array}$ \\
\hline $\begin{array}{l}\text { Floer, } 2014 \\
{[28]}\end{array}$ & HD ECU & $\begin{array}{l}248 \\
252\end{array}$ & $\begin{array}{l}243 \\
249\end{array}$ & $\begin{array}{l}63 \\
64\end{array}$ & $\begin{array}{l}44.9 \% \\
49.0 \%\end{array}$ & $\begin{array}{l}\text { S: NR } \\
\text { D: NR }\end{array}$ & $\begin{array}{l}\text { S: NR } \\
\text { D: NR }\end{array}$ & $\begin{array}{l}\text { Olympus CF-H180Al/AL, } \\
\text { Fujifilm EC-590 WM4 / } \\
\text { WL4 }\end{array}$ \\
\hline $\begin{array}{l}\text { Van Doorne, } \\
2017 \text { [31] }\end{array}$ & HD ECU & $\begin{array}{l}533 \\
530\end{array}$ & $\begin{array}{l}514 \\
486\end{array}$ & $\begin{array}{l}65 \\
65\end{array}$ & $\begin{array}{l}53.5 \% \\
49.8 \%\end{array}$ & $\begin{array}{l}\text { S: } 51.8 \% \\
\text { D: } 48.2 \%\end{array}$ & $\begin{array}{l}\text { S: } 51.9 \% \\
\text { D: } 48.1 \%\end{array}$ & $\begin{array}{l}\text { Olympus CF-H180AL, } \\
\text { CF-HQ190 L, PCF- } \\
\text { H180AL, Fujinon EC } \\
\text { 350-WL }\end{array}$ \\
\hline $\begin{array}{l}\text { Wada, } 2018 \\
{[32]}\end{array}$ & HD ECU & $\begin{array}{l}238 \\
239\end{array}$ & $\begin{array}{l}237 \\
235\end{array}$ & $\begin{array}{l}62.2 \\
61.2\end{array}$ & $\begin{array}{l}48.3 \% \\
51.0 \%\end{array}$ & $\begin{array}{l}\text { S: } 58.0 \% \\
\text { D: } 42.0 \%\end{array}$ & $\begin{array}{l}\text { S: } 58.6 \% \\
\text { D: } 41.4 \%\end{array}$ & $\begin{array}{l}\text { Olympus PCF-H290ZI, } \\
\text { CF-HQ290, CF-HQ290ZI }\end{array}$ \\
\hline $\begin{array}{l}\operatorname{Rex}(1), 2018 \\
{[34]}\end{array}$ & HD ECU & $\begin{array}{l}316 \\
316\end{array}$ & $\begin{array}{l}295 \\
299\end{array}$ & $\begin{array}{l}62.6 \\
63.2\end{array}$ & $\begin{array}{l}52.9 \% \\
52.8 \%\end{array}$ & $\begin{array}{l}\text { S: } 94.6 \% \\
\text { D: } 5.4 \%\end{array}$ & $\begin{array}{l}\text { S: } 92.3 \% \\
\text { D: } 7.7 \%\end{array}$ & $\begin{array}{l}\text { Olympus } \mathrm{H} 190, \mathrm{H} 180 \\
\text { series }\end{array}$ \\
\hline $\begin{array}{l}\text { Bhattacharyya, } \\
2017 \text { [25] }\end{array}$ & HD ECV & $\begin{array}{l}267 \\
267\end{array}$ & $\begin{array}{l}265 \\
266\end{array}$ & $\begin{array}{l}67 \\
68\end{array}$ & $\begin{array}{l}67.9 \% \\
60.9 \%\end{array}$ & $\begin{array}{l}\text { S: } 100 \% \\
\text { D: } 0 \%\end{array}$ & $\begin{array}{l}\text { S: } 100 \% \\
\text { D: } 0 \%\end{array}$ & Olympus CF-H260 \\
\hline $\begin{array}{l}\text { Jacob, } 2019 \\
{[29]}\end{array}$ & HD ECV & $\begin{array}{l}146 \\
214\end{array}$ & $\begin{array}{l}138 \\
182\end{array}$ & $\begin{array}{l}\text { NR } \\
\text { NR }\end{array}$ & $\begin{array}{l}50.7 \% \\
56.7 \%\end{array}$ & $\begin{array}{l}\text { S: NR } \\
\text { D: NR }\end{array}$ & $\begin{array}{l}\text { S: NR } \\
\text { D: NR }\end{array}$ & $\begin{array}{l}\text { Olympus CF-190 L/I, CF- } \\
\text { H180 L/I, PCF-H180AL/I, } \\
\text { PCF-H190 L/I }\end{array}$ \\
\hline Ngu, 2017 [15] & HD ECV & $\begin{array}{l}884 \\
888\end{array}$ & $\begin{array}{l}884 \\
888\end{array}$ & $\begin{array}{l}62.1 \\
61.7\end{array}$ & $\begin{array}{l}56.8 \% \\
57.1 \%\end{array}$ & $\begin{array}{l}\text { S: } 45.6 \% \\
\text { D: } 54.4 \%\end{array}$ & $\begin{array}{l}\text { S: } 44.4 \% \\
\text { D: } 55.6 \%\end{array}$ & $\begin{array}{l}\text { Olympus (details not } \\
\text { reported) }\end{array}$ \\
\hline $\begin{array}{l}\operatorname{Rex}(2), 2019 \\
{[30]}\end{array}$ & HD ECV & $\begin{array}{r}99 \\
101\end{array}$ & $\begin{array}{r}99 \\
101\end{array}$ & $\begin{array}{l}61.7 \\
62.7\end{array}$ & $\begin{array}{l}42.4 \% \\
56.4 \%\end{array}$ & $\begin{array}{l}\text { S:NR } \\
\text { D: NR }\end{array}$ & $\begin{array}{l}\text { S: NR } \\
\text { D: NR }\end{array}$ & $\begin{array}{l}\text { Olympus (details not } \\
\text { reported) }\end{array}$ \\
\hline $\begin{array}{l}\text { Von Figura, } \\
2019 \text { [33] }\end{array}$ & HD ECV & $\begin{array}{l}125 \\
125\end{array}$ & $\begin{array}{l}122 \\
118\end{array}$ & $\begin{array}{l}65.3 \\
63.6\end{array}$ & $\begin{array}{l}62.9 \% \\
51.7 \%\end{array}$ & $\begin{array}{l}\text { S: } 38.5 \% \\
\text { D: } 61.5 \%\end{array}$ & $\begin{array}{l}\text { S: } 45.8 \% \\
\text { D: } 54.2 \%\end{array}$ & Olympus CF-HQ190 \\
\hline $\begin{array}{l}\text { Karsenti, } 2020 \\
\text { [35] }\end{array}$ & HD ECV & $\begin{array}{l}1032 \\
1032\end{array}$ & $\begin{array}{l}1032 \\
1026\end{array}$ & $\begin{array}{l}57.4 \\
59.3\end{array}$ & $\begin{array}{l}48.9 \% \\
47.4 \%\end{array}$ & $\begin{array}{l}\text { S: NR } \\
\text { D: NR }\end{array}$ & $\begin{array}{l}\text { S: NR } \\
\text { D: NR }\end{array}$ & $\begin{array}{l}\text { Olympus CF-H190, Fuji- } \\
\text { film EC-690 WM/EC-600 } \\
\text { WM }\end{array}$ \\
\hline
\end{tabular}

ECU colonoscopy compared to HD statistically and clinically (65.6\% vs $52.9 \%$, RR: $1.26,95 \%$ Cl $1.09-1.46, P=0.002, \mathrm{I}^{2}=$ $74.9 \%$ ) ( Fig. 2d) [26, 28,32,34].

Three studies compared ECU to HD colonoscopy and increased MPP was noted for ECU statistically and clinically (MD: 0.53, 95\% Cl 0.39-0.67, $P<0.001, I^{2}=0 \%$ ) (Supplementary Fig. 1a) [26, 28,32]. No clinical and statistically significant difference in MPP was observed for ECV group compared to HD (MD:-0.13, 95\% Cl-0.31-0.05, $P=0.16, \mathrm{I}^{2}=4.4 \%$ ) (Supplementary Fig. 1b) [25, 29,33]. Four studies assessed MAP for ECU and HD colonoscopy and improved MAP was noted for ECU group clinically and statistically (MD: $0.31,95 \% \mathrm{Cl} 0.19-$ $0.44, P<0.001, I^{2}=0 \%$ ) (Supplementary Fig. 1c) $[28,31,32$, 34]. The MAP was not clinically and statistically different for ECV group when compared to HD colonoscopy (MD: 0.14, $95 \%$
Cl-0.01-0.28, $\quad \mathrm{p}=0.06, \mathrm{I}^{2}=53.1 \%$ ) (Supplementary Fig. 1d) $[15,23,28,35]$.

\section{Network meta-analysis}

The results for network meta-analysis are summarized in table 3 and demonstrated using network forest plots ( $\mathbf{F i g . 3 a}$, $>$ Fig.3b, $>$ Fig.3c, $>$ Fig.3d, $>$ Fig.3e, $>$ Fig.3f, $>$ Fig.3g, - Fig.3h). Network diagrams for respective forest plots are shown in Supplementary Fig.2. Overall improved PDR was seen with ECU compared to HD colonoscopy (RR: $1.25,95 \% \mathrm{Cl}$ $1.10-1.43$ ) as well as ECV compared to HD colonoscopy (RR: 1.14, $95 \%$ Cl 1.02-1.29). Improved ADR was noted for ECU colonoscopy compared to HD colonoscopy (RR: $1.22,95 \% \mathrm{Cl}$ : 1.06-1.40). These results were both statistically and clinically significant. ADR improvement was not statistically significant 
- Table 2 Outcomes for individual studies.

\begin{tabular}{|c|c|c|c|c|c|c|c|c|c|}
\hline \multirow[t]{2}{*}{ Study, year } & \multirow{2}{*}{$\begin{array}{l}\text { Tech- } \\
\text { niques } \\
\text { compared }\end{array}$} & \multicolumn{8}{|c|}{ Outcomes } \\
\hline & & $\begin{array}{l}\text { Total } \\
\text { Polyps, n }\end{array}$ & $\begin{array}{l}\text { Total Ade- } \\
\text { nomas, n }\end{array}$ & ADR, n (\%) & PDR, n (\%) & AADR, n (\%) & SADR, n (\%) & NDR, n (\%) & CIR, n (\%) \\
\hline $\begin{array}{l}\text { Biecker, } \\
2015 \text { [26] }\end{array}$ & HD ECU & $\begin{array}{l}246 \\
388\end{array}$ & $\begin{array}{l}\text { NR } \\
\text { NR }\end{array}$ & $\begin{array}{l}69(27.7 \%) \\
87(36.3 \%)\end{array}$ & $\begin{array}{l}106(42.6 \%) \\
138(57.5 \%)\end{array}$ & $\begin{array}{l}\text { NR } \\
\text { NR }\end{array}$ & $\begin{array}{l}\text { NR } \\
\text { NR }\end{array}$ & $\begin{array}{l}\text { NR } \\
\text { NR }\end{array}$ & $\begin{array}{l}N R \\
N R\end{array}$ \\
\hline $\begin{array}{l}\text { De Palma, } \\
2018 \text { [27] }\end{array}$ & HD ECU & $\begin{array}{l}\text { NR } \\
\text { NR }\end{array}$ & $\begin{array}{l}129 \\
176\end{array}$ & $\begin{array}{l}39(28.5 \%) \\
38(27.7 \%)\end{array}$ & $\begin{array}{l}\text { NR } \\
\text { NR }\end{array}$ & $\begin{array}{l}\text { NR } \\
\text { NR }\end{array}$ & $\begin{array}{l}\text { NR } \\
\text { NR }\end{array}$ & $\begin{array}{l}\text { NR } \\
\text { NR }\end{array}$ & $\begin{array}{l}\text { NR } \\
\text { NR }\end{array}$ \\
\hline $\begin{array}{l}\text { Floer, } 2014 \\
\text { [28] }\end{array}$ & HD ECU & $\begin{array}{l}189 \\
312\end{array}$ & $\begin{array}{l}88 \\
144\end{array}$ & $\begin{array}{l}50(20.6 \%) \\
87(34.9 \%)\end{array}$ & $\begin{array}{l}93(38.3 \%) \\
138(55.4 \%)\end{array}$ & $\begin{array}{l}\text { NR } \\
\text { NR }\end{array}$ & $\begin{array}{l}\text { NR } \\
\text { NR }\end{array}$ & $\begin{array}{l}\text { NR } \\
\text { NR }\end{array}$ & $\begin{array}{l}229(94.2 \%) \\
238(95.6 \%)\end{array}$ \\
\hline $\begin{array}{l}\text { Van Doorne, } \\
2017 \text { [31] }\end{array}$ & HD ECU & $\begin{array}{l}883 \\
982\end{array}$ & $\begin{array}{l}610 \\
700\end{array}$ & $\begin{array}{l}271(52.7 \%) \\
260(53.5 \%)\end{array}$ & $\begin{array}{l}\text { NR } \\
\text { NR }\end{array}$ & $\begin{array}{l}111(21.6 \%) \\
103(21.2 \%)\end{array}$ & $\begin{array}{l}\text { NR } \\
\text { NR }\end{array}$ & $\begin{array}{l}\text { NR } \\
\text { NR }\end{array}$ & $\begin{array}{l}518(97.2 \%) \\
508(96.0 \%)\end{array}$ \\
\hline $\begin{array}{l}\text { Wada, } 2018 \\
\text { [32] }\end{array}$ & HD ECU & $\begin{array}{l}197 \\
312\end{array}$ & $\begin{array}{l}158 \\
263\end{array}$ & $\begin{array}{l}93(39.2 \%) \\
132(56.2 \%)\end{array}$ & $\begin{array}{l}117(49.4 \%) \\
148(63.0 \%)\end{array}$ & $\begin{array}{l}11(4.6 \%) \\
18(7.7 \%)\end{array}$ & $\begin{array}{l}\text { NR } \\
\text { NR }\end{array}$ & $\begin{array}{l}7(3.0 \%) \\
5(2.1 \%)\end{array}$ & $\begin{array}{l}237(99.6 \%) \\
235(98.3 \%)\end{array}$ \\
\hline $\begin{array}{l}\operatorname{Rex}(1) \\
2018 \text { [34] }\end{array}$ & HD ECU & $\begin{array}{l}\text { NR } \\
\text { NR }\end{array}$ & $\begin{array}{l}445 \\
543\end{array}$ & $\begin{array}{l}166(56.3 \%) \\
191(63.9 \%)\end{array}$ & $\begin{array}{l}226(76.6 \%) \\
247(82.6 \%)\end{array}$ & $\begin{array}{l}\text { NR } \\
\text { NR }\end{array}$ & $\begin{array}{l}36(12.2 \%) \\
33(11.0 \%)\end{array}$ & $\begin{array}{l}\text { NR } \\
\text { NR }\end{array}$ & $\begin{array}{l}295(100.0 \%) \\
299(100.0 \%)\end{array}$ \\
\hline $\begin{array}{l}\text { Bhattachar- } \\
\text { yya, } 2017 \\
{[25]}\end{array}$ & HD ECV & $\begin{array}{l}470 \\
436\end{array}$ & $\begin{array}{l}359 \\
336\end{array}$ & $\begin{array}{l}167(63.0 \%) \\
162(60.9 \%)\end{array}$ & $\begin{array}{l}185(69.8 \%) \\
187(70.3 \%)\end{array}$ & $\begin{array}{l}49(18.5 \%) \\
45(16.9 \%)\end{array}$ & $\begin{array}{l}\mathrm{NR} \\
\mathrm{NR}\end{array}$ & $\begin{array}{l}15(5.7 \%) \\
14(5.3 \%)\end{array}$ & $\begin{array}{l}N R \\
N R\end{array}$ \\
\hline $\begin{array}{l}\text { Jacob, } 2019 \\
\text { [29] }\end{array}$ & HD ECV & $\begin{array}{l}\text { NR } \\
\text { NR }\end{array}$ & $\begin{array}{l}\text { NR } \\
\text { NR }\end{array}$ & $\begin{array}{l}40(29.0 \%) \\
67(36.8 \%)\end{array}$ & $\begin{array}{l}48(34.8 \%) \\
97(53.3 \%)\end{array}$ & $\begin{array}{l}\text { NR } \\
\text { NR }\end{array}$ & $\begin{array}{l}\text { NR } \\
\text { NR }\end{array}$ & $\begin{array}{l}\text { NR } \\
\text { NR }\end{array}$ & $\begin{array}{l}\text { NR } \\
\text { NR }\end{array}$ \\
\hline $\begin{array}{l}\text { Ngu, } 2017 \\
{[15]}\end{array}$ & HD ECV & $\begin{array}{l}\text { NR } \\
\text { NR }\end{array}$ & $\begin{array}{l}\text { NR } \\
\text { NR }\end{array}$ & $\begin{array}{l}320(36.2 \%) \\
362(40.8 \%)\end{array}$ & $\begin{array}{l}424(50.0 \%) \\
480(54.1 \%)\end{array}$ & $\begin{array}{l}\text { NR } \\
\text { NR }\end{array}$ & $\begin{array}{l}10(1.1 \%) \\
20(2.3 \%)\end{array}$ & $\begin{array}{l}20(2.3 \%) \\
36(4.1 \%)\end{array}$ & $\begin{array}{l}852(95.9 \%) \\
858(96.6 \%)\end{array}$ \\
\hline $\begin{array}{l}\operatorname{Rex}(2), \\
2019[30]\end{array}$ & HD ECV & $\begin{array}{l}\text { NR } \\
\text { NR }\end{array}$ & $\begin{array}{l}\text { NR } \\
\text { NR }\end{array}$ & $\begin{array}{l}52(52.5 \%) \\
62(61.4 \%)\end{array}$ & $\begin{array}{l}\text { NR } \\
\text { NR }\end{array}$ & $\begin{array}{l}\text { NR } \\
\text { NR }\end{array}$ & $\begin{array}{l}11(11.1 \%) \\
20(19.8 \%)\end{array}$ & $\begin{array}{l}\text { NR } \\
\text { NR }\end{array}$ & $\begin{array}{l}99(100.0 \%) \\
101(100.0 \%)\end{array}$ \\
\hline $\begin{array}{l}\text { Von Figura, } \\
2019 \text { [33] }\end{array}$ & HD ECV & $\begin{array}{l}211 \\
169\end{array}$ & $\begin{array}{l}97 \\
87\end{array}$ & $\begin{array}{l}52(42.6 \%) \\
45(38.1 \%)\end{array}$ & $\begin{array}{l}64(52.5 \%) \\
64(54.2 \%)\end{array}$ & $\begin{array}{l}\text { NR } \\
\text { NR }\end{array}$ & $\begin{array}{l}N R \\
N R\end{array}$ & $\begin{array}{l}\text { NR } \\
\text { NR }\end{array}$ & $\begin{array}{l}122(100.0 \%) \\
118(100.0 \%)\end{array}$ \\
\hline $\begin{array}{l}\text { Karsenti, } \\
2020 \text { [35] }\end{array}$ & HD ECV & $\begin{array}{l}\text { NR } \\
\text { NR }\end{array}$ & $\begin{array}{l}557 \\
800\end{array}$ & $\begin{array}{l}304(29.4 \%) \\
402(39.2 \%)\end{array}$ & $\begin{array}{l}389(37.7 \%) \\
474(46.2 \%)\end{array}$ & $\begin{array}{l}95(9.2 \%) \\
114(11.1 \%)\end{array}$ & $\begin{array}{l}\text { NR } \\
\text { NR }\end{array}$ & $\begin{array}{l}\text { NR } \\
\text { NR }\end{array}$ & $\begin{array}{l}\text { NR } \\
\text { NR }\end{array}$ \\
\hline
\end{tabular}

for ECV colonoscopy compared to HD colonoscopy (RR: 1.12, $95 \% \mathrm{Cl}$ : 0.98-1.29). No statistically significant difference was noted between ECV and ECU colonoscopy in terms of ADR, PDR, NDR, AADR, CIR and MAP. ECV was found to have statistically higher SADR compared to both HD (RR: $1.87,95 \% \mathrm{Cl}$ 1.13-3.11) and ECU (RR: 2.07, $95 \%$ Cl 1.06-4.06) colonoscopy. Lower MPP was noted with ECV group compared to ECU colonoscopy (MD:-0.65, $95 \% \mathrm{Cl}-0.87--0.43$ ). This was both statistically and clinically significant.

A subgroup analysis was performed for low detectors using control $A D R$ of $\leq 40 \%$ and $\leq 30 \%$ as reference. The ADR for both ECV and ECU showed improvement for endoscopist when control ADR was $\leq 40 \%$ (RR: 1.22 and RR: 1.37, respectively for $E C V$ and $E C U$ ) and $\leq 30 \%$ (RR: 1.31 and RR: 1.33, respectively for ECV and ECU) ( Table 3 ).

\section{Adverse events}

Lower rates of overall complications (RR: 0.20 , $95 \% \mathrm{Cl} 0.08-$ 0.50 ) and particularly lacerations/erosions (RR: $0.12,95 \% \mathrm{Cl}$ 0.05-0.29) were noted with HD colonoscopy compared to ECU colonoscopy. Similarly, lower overall complication rates (RR: $0.14,95 \% \mathrm{Cl} 0.02-0.84)$ and particularly lacerations/erosions (RR: $0.11,95 \% \mathrm{Cl}$ 0.02-0.70) were also noted for ECV colonoscopy when compared to ECU colonoscopy. The adverse events are compared and summarized in Supplementary Table 2.

\section{Ranking of interventions}

Based on frequentist approach, ECU was better compared to ECV in terms of PDR, ADR, AADR, MAP and MPP. ECV was found to be better compared to ECU in terms of NDR, SADR, and lower rates for complications (overall, lacerations, perforations, and major bleeding) (Supplementary Fig. 3). 


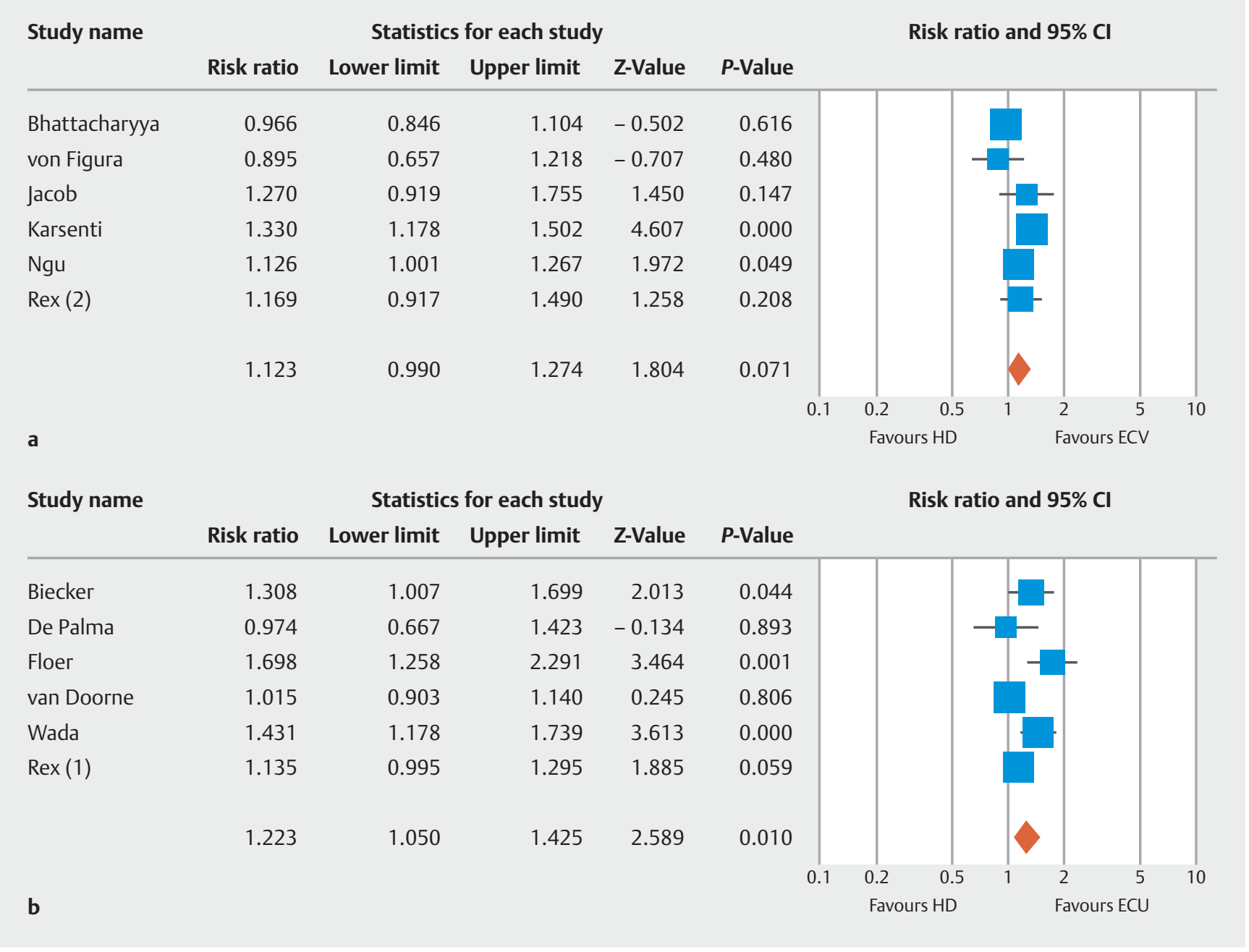

- Fig. 2a, b Forest plot comparing a ADR for ECV vs HD, b ADR for ECU vs HD. ADR, adenoma detection rate; $\mathrm{Cl}$, confidence interval; ECU, first-generation Endocuff colonoscopy; ECV, Endocuff Vision colonoscopy; HD, high-definition colonoscopy; PDR, polyp detection rate.

\section{Risk of bias/inconsistency}

The visual assessment of funnel plot based on ADR for all studies revealed visible symmetry (Supplementary Fig.4). No significant publication bias was observed based on Egger's regression analysis $(P=0.47)$. The risk of bias for individual studies are highlighted in Supplementary Table 3. High risk of bias was observed for all studies as the endoscopists were not blinded to intervention groups as well as outcomes. Inconsistency between direct and indirect evidence was not applicable due to lack of number of study designs.

\section{Certainty of evidence}

The evidence based on direct meta-analysis for all outcomes assessed was MODERATE. The evidence was downgraded due to high risk of bias in the included studies. The evidence based on network meta-analysis for outcomes (ADR, PDR, MAP, MPP, and CIR) assessed was LOW. The evidence was rated down due to indirectness of results and high risk of study bias. Evidence for other outcomes (SADR, NDR, and AADR) based on network meta-analysis was rated VERY LOW. The evidence was rated down due to indirectness of results, high risk of study bias, and imprecision due to low number of studies.

\section{Discussion}

The results of our direct and network meta-analysis suggest the relative lack of efficacy of ECV in terms of ADR, AADR, NDR, MAP and MPP when compared to HD colonoscopy. Significantly lower MPP was observed when ECV was compared to ECU using the network meta-analysis model.

ADR is a well-established colonoscopy quality indicator and has been inversely linked with incidence CRC (i. e. increase ADR leading to decrease $C R C)[5,36]$. Corley et al. demonstrated a reduction of CRC mortality by $5 \%$ for every $1 \%$ increment in ADR [5]. Add-on devices (such as Endocap, Endocuff, EndoRing, G-EYE, AmplifEYE) are thought to assist with increasing ADR by flattening the mucosal fold on withdrawal leading to a thorough examination [14]. Evidence of increased ADR was observed with original ECU compared to HD colonoscopy based on previous RCTs and current meta-analysis, the device was re- 


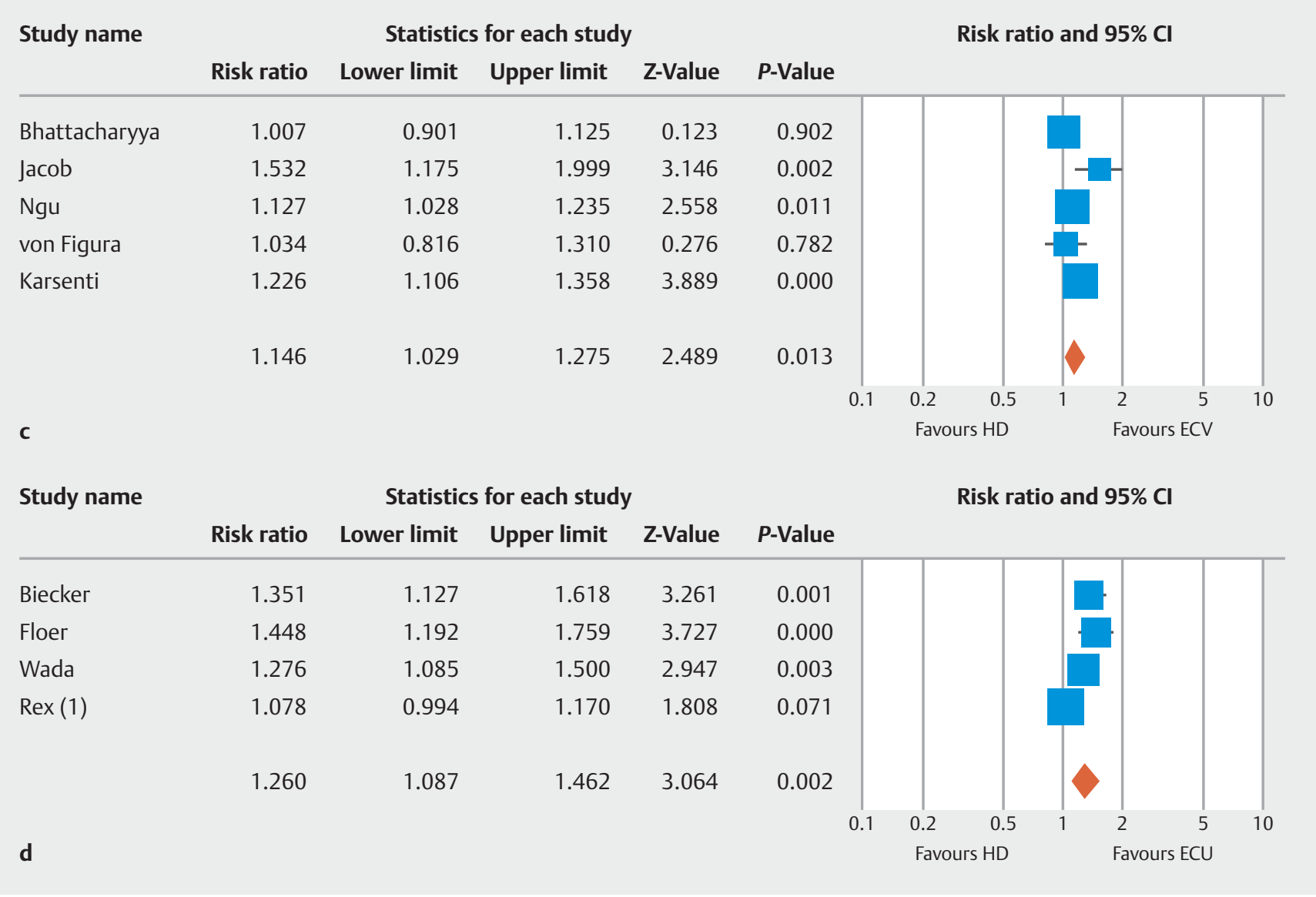

- Fig. 2 c, d Forest plot comparing c PDR for ECV vs HD, and d PDR for ECU vs HD. ADR, adenoma detection rate; Cl, confidence interval; ECU, first-generation Endocuff colonoscopy; ECV, Endocuff Vision colonoscopy; HD, high-definition colonoscopy; PDR, polyp detection rate.

placed with the newer ECV due to significant adverse events particularly lacerations and erosions [27,32]. There was also the lack of efficacy of improving overall MAP and MPP using ECV in addition to ADR. Although the safety profile of ECV was similar to HD colonoscopy and significantly better compared to $E C U$, the relative lack of improved efficacy in terms of major outcome metrics such as ADR, MAP and MPP compared to HD colonoscopy makes the device less cost effective. However, the use of ECV maybe justified for low detectors as we demonstrated significantly improved ADR when control ADR for endoscopist was $\leq 30 \%$ and $\leq 40 \%$ respectively.

Recently, there has been interest in improving the SADR [37, 38]. The culprit lesions, i.e. SSAs/polyps and traditional serrated adenomas, are attributed to interval CRC through the serrated adenoma pathway [3]. Interestingly, we found significantly improved SADR using ECV compared with HD colonoscopy (RR: 1.87, $95 \% \mathrm{Cl} 1.13-3.11$ ) as well as ECU colonoscopy (RR: 2.07, $95 \% \mathrm{Cl} 1.06-4.06)$. These results are limited as only three studies ( 1 comparing ECU to HD and 2 comparing ECV to HD) evaluated this effect.

Previous meta-analyses demonstrated significant improvement in ADR using Endocuff colonoscopy. Williet et al. (RR: $1.20,95 \% \mathrm{Cl} 1.06-1.36)$, Facciorusso et al. (Odds ratio (OR): $1.22,95 \% \mathrm{Cl} 1.04-1.44$ ), Chin et al. (OR: 1.49 , $95 \% \mathrm{Cl} 1.23-$
1.80), Jian et al. (OR: $1.37,95 \% \mathrm{Cl} 1.15-1.62$ ), and Triantafyllou et al. (RR: 1.18, $95 \% \mathrm{Cl} 1.05-1.32$ ) showed improved detection of adenoma using Endocuff colonoscopy compared to HD colonoscopy. These analyses have serious limitation as all the Endocuff devices (ECU and ECV) were pooled together to demonstrate significantly improved colonoscopy outcome metrics [7, 16-19]. Although somewhat similar, these devices are still different and should be evaluated as such. Further, these metaanalyses included RCTs published as abstracts or conference proceedings which somewhat limit the validity of the overall results as it is hard to assess the study quality based on abstracts alone $[7,16]$. As newer RCTs were published, we excluded the abstracts to generate the best evidence and provided separate analysis for each device to help guide the clinician regarding the utility and efficacy of these devices.

An important observation to note here is the improvement in ADR for low detectors using both ECU and ECV compared with HD. The use of Endocuff can perhaps be justified for low detectors as is evident from the results.

Our meta-analysis is not without limitations. The biggest one was the lack of studies directly comparing ECU with ECV. Another important limitation worth mentioning was that majority of the studies included patients other than for a screening indication. This limits the overall conclusion as "true ADR" 


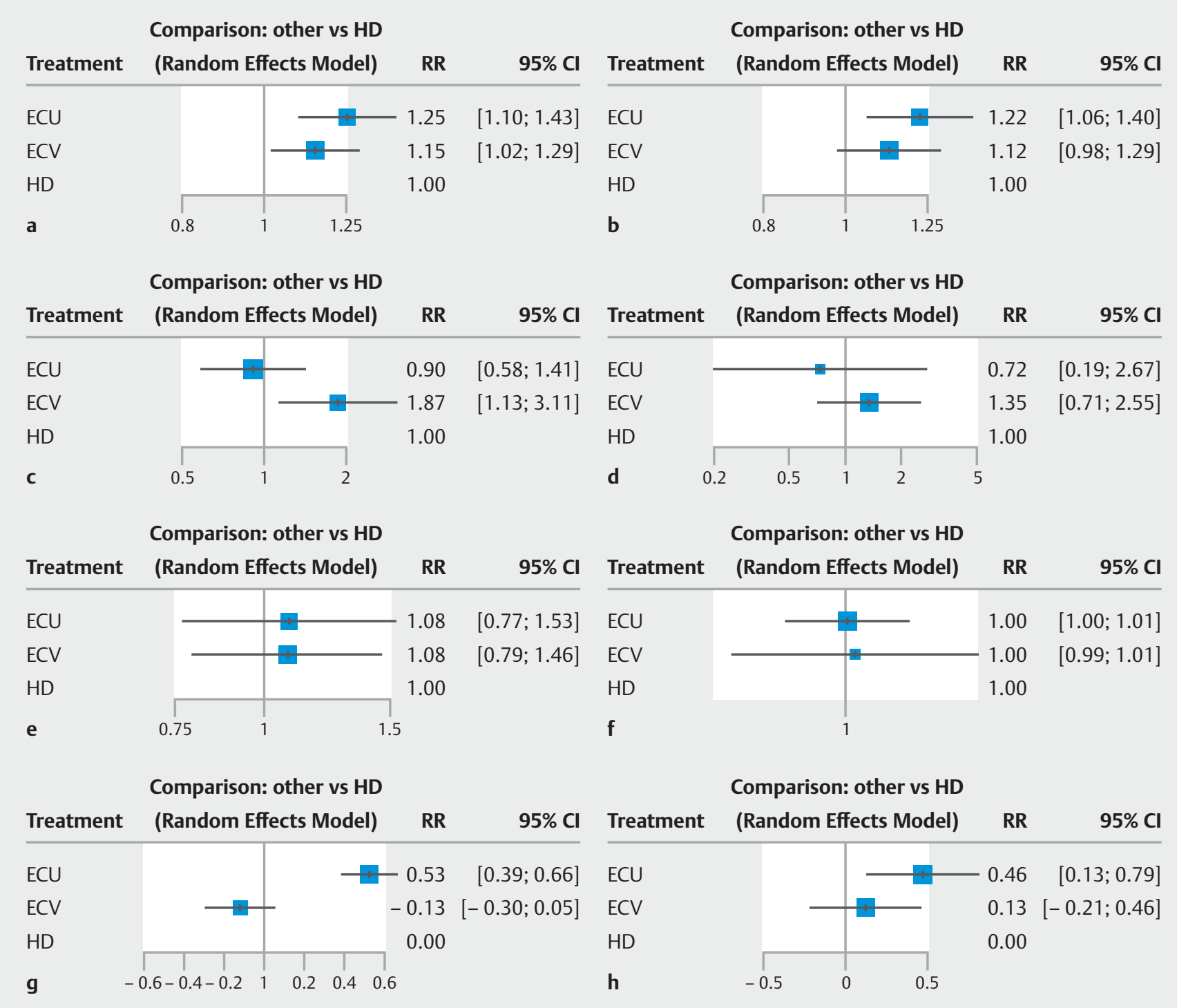

Fig. 3 Forest plots for network meta-analysis a PDR, b ADR, c SADR, d NDR, e AADR, f CIR, g MPP, and h MAP. AADR, advanced adenoma detection rate; ADR, adenoma detection rate; CIR, cecal intubation rate; ECU, first-generation Endocuff; ECV, Endocuff Vision; HD, high-definition colonoscopy; MAP, mean adenoma per procedure; MPP, mean polyp per procedure; NDR, neoplasia detection rate; PDR, polyp detection rate. Note: Line represents a direct comparison, width of line corresponds to weight, overall effect and number of studies).

should be accounted for screening colonoscopies, and hence our results should be interpreted keeping in mind this limitation [39]. A high risk of bias was noted as it is virtually impossible to blind the endoscopist to treatment allocation and outcome assessment. This bring into play performance and detection biases. Finally, factors related to the endoscopist (training level, experience, fatigue factor) and patient (family history, ethnicity, bowel preparation) can have an influence on the outcome of colonoscopy and were not accounted in the current study. This may account for the heterogeneity seen in our study.

Despite these limitations, we included RCTs with a robust number of study population. We provided both direct and indirect evidence regarding the outcomes of particular intervention. We further provided subgroup analysis result based on control ADR of the studies to assess if these devices had impact on low detectors.

\section{Conclusions}

In conclusion, the utility of ECV is somewhat limited in terms of improving the outcome metrics, including ADR, AADR, NDR, MAP and MPP. Although improved SADR was noted, that is limited due to the low number of studies reporting this outcome. ECV can perhaps be best justified for low detectors to improve ADR and PDR. Further device modification is needed to improve outcome metrics as well as keeping the safety profile as demonstrated by ECV. 
- Table 3 Outcomes based on network meta-analysis

\begin{tabular}{|c|c|c|c|c|c|}
\hline Study outcome & No. of Studies compared & ECU vs HD & ECV vs HD & ECV vs ECU & \\
\hline & & \multicolumn{3}{|l|}{ Relative risk (95\% Cl) } & $\mathrm{I}^{2}$ \\
\hline Overall PDR & 9 & $1.25(1.10-1.43)^{1}$ & $1.14(1.02-1.29)^{1}$ & $0.92(0.77-1.09)$ & $70.9 \%$ \\
\hline $\mathrm{PDR}^{2}$ & 3 & $1.08(0.99-1.17)$ & $1.01(0.91-1.12)$ & $0.94(0.82-1.07)$ & $0 \%$ \\
\hline $\mathrm{PDR}^{3}$ & 6 & $1.35(1.20-1.52)^{1}$ & $1.21(1.10-1.33)^{1}$ & $0.90(0.77-1.04)$ & $34.5 \%$ \\
\hline $\mathrm{PDR}^{4}$ & 5 & $1.14(1.01-1.29)^{1}$ & $1.06(0.96-1.18)$ & $0.93(0.80-1.09)$ & $47.8 \%$ \\
\hline $\mathrm{PDR}^{5}$ & 4 & $1.40(1.20-1.63)^{1}$ & $1.29(1.13-1.47)^{1}$ & $0.92(0.75-1.13)$ & $23.7 \%$ \\
\hline Overall ADR & 12 & $1.22(1.06-1.40)^{1}$ & $1.12(0.98-1.29)$ & $0.92(0.76-1.12)$ & $69.2 \%$ \\
\hline $\mathrm{ADR}^{2}$ & 5 & $1.07(0.96-1.19)$ & $1.00(0.88-1.13)$ & $0.93(0.79-1.10)$ & $22.8 \%$ \\
\hline $\mathrm{ADR}^{3}$ & 7 & $1.37(1.16-1.61)^{1}$ & $1.22(1.07-1.42)^{1}$ & $0.90(0.72-1.12)$ & $45.3 \%$ \\
\hline $\mathrm{ADR}^{4}$ & 7 & $1.16(0.99-1.34)$ & $1.05(0.91-1.20)$ & $0.90(0.74-1.11)$ & $63.0 \%$ \\
\hline $\mathrm{ADR}^{5}$ & 5 & $1.33(1.06-1.67)^{1}$ & $1.31(1.05-1.64)^{1}$ & $0.99(0.72-1.36)$ & $42.5 \%$ \\
\hline AADR & 4 & $1.09(0.77-1.53)$ & $1.08(0.79-1.46)$ & $0.99(0.63-1.58)$ & $38.1 \%$ \\
\hline SADR & 3 & $0.90(0.58-1.41)$ & $1.87(1.13-3.11)^{1}$ & $2.07(1.06-4.06)^{1}$ & $0 \%$ \\
\hline NDR & 3 & $0.72(0.19-2.67)$ & $1.34(0.71-2.55)$ & $1.87(0.44-8.02)$ & $52.1 \%$ \\
\hline \multirow[t]{2}{*}{$\mathrm{CIR}$} & 7 & $1.00(0.99-1.01)$ & $1.00(0.99-1.01)$ & $1.00(0.99-1.01)$ & $0 \%$ \\
\hline & & \multicolumn{3}{|c|}{ Mean difference $(95 \% \mathrm{Cl})$} & \\
\hline Overall MPP & 6 & $0.53(0.39-0.66)^{1}$ & $-0.13(-0.30-0.05)$ & $-0.65(-0.87--0.43)^{1}$ & $0 \%$ \\
\hline Overall MAP & 9 & $0.46(0.13-0.79)^{1}$ & $0.12(-0.21-0.46)$ & $-0.34(-0.81-0.13)$ & $92.5 \%$ \\
\hline \multicolumn{6}{|c|}{$\begin{array}{l}\text { AADR, advanced adenoma detection rate; } \\
\text { copy; ECV, Endocuff Vision colonoscopy; } \mathrm{H} \\
\text { detection rate; PDR, polyp detection rate; } \\
{ }^{1} \text { P }<0.05 \text { for the study estimate } \\
{ }^{2} \text { Denotes studies with control ADR }>40 \% \\
{ }^{3} \text { Denotes studies with control ADR } \leq 40 \% \\
{ }^{4} \text { Denotes studies with control ADR }>30 \% \\
{ }^{5} \text { Denotes studies with control ADR } \leq 30 \%\end{array}$} \\
\hline
\end{tabular}

Competing interests

The authors declare that they have no conflict of interest.

\section{References}

[1] Allemani C, Rachet B, Weir HK et al. Colorectal cancer survival in the USA and Europe: a CONCORD high-resolution study. BMJ open 2013; 3: e003055

[2] Cancer of the Colon and Rectum - Cancer Stat Facts. (n.d.). Available at (Accessed on March 11, 2020): https://seer.cancer.gov/statfacts/ html/colorect.html

[3] Leggett B, Whitehall V. Role of the serrated pathway in colorectal cancer pathogenesis. Gastroenterology 2010; 138: 2088-2100

[4] Rex DK, Petrini JL, Baron TH et al. Quality indicators for colonoscopy. Gastrointest Endosc 2006; 63: S16-S28

[5] Corley DA, Jensen CD, Marks AR et al. Adenoma detection rate and risk of colorectal cancer and death. N Engl J Med 2014; 370: 12981306
[6] Rex DK, Schoenfeld PS, Cohen J et al. Quality indicators for colonoscopy. Gastrointest Endosc 2015; 81: 31-53

[7] Facciorusso A, Triantafyllou K, Murad MH et al. Compared abilities of endoscopic techniques to increase colon adenoma detection rates: a network meta-analysis. Clin Gastroenterol Hepatol 2019; 17: 24392454.e25

[8] Aziz M, Sharma S, Fatima R et al. How to increase proximal adenoma detection rate: a meta-analysis comparing water exchange, water immersion and air/CO2 insufflation methods for colonoscopy. Ann Gastroenterol 2020; 33: 178-186

[9] Fuccio L, Frazzoni L, Hassan C et al. Water exchange colonoscopy increases adenoma detection rate: a systematic review with network meta-analysis of randomized controlled studies. Gastrointest Endosc 2018; 88: 589-597.e11

[10] Aziz M, Weissman S, Khan Z et al. Use of 2 observers increases adenoma detection rate during colonoscopy: systematic review and meta-analysis. Clin Gastroenterol Hepatol 2019: pii: S1542-3565(19) 30777-3

[11] Aziz M, Sharma S, Ghazaleh S et al. The anti-spasmodic effect of peppermint oil during colonoscopy: a systematic review and meta-analysis. Minerva Gastroenterol Dietol 2020: doi:10.23736/S1121$421 \times .20 .02652-5$ 
[12] Desai M, Bilal M, Hamade N et al. Increasing adenoma detection rates in the right side of the colon comparing retroflexion with a second forward view: a systematic review. Gastrointest Endosc 2019; 89: 453-459.e3

[13] Aziz M, Fatima R, Dong C et al. The impact of deep convolutional neural network-based artificial intelligence on colonoscopy outcomes: A systematic review with meta-analysis. J Gastroenterol Hepatol 2020: doi:10.1111/jgh.15070

[14] Gkolfakis P, Tziatzios G, Spartalis E et al. Colonoscopy attachments for the detection of precancerous lesions during colonoscopy: A review of the literature. World J Gastroenterol 2018; 24: 4243-4253

[15] Ngu WS, Bevan R, Tsiamoulos ZP et al. Improved adenoma detection with Endocuff Vision: the ADENOMA randomised controlled trial. Gut 2019; 68: 280-288

[16] Williet N, Tournier Q, Vernet C et al. Effect of Endocuff-assisted colonoscopy on adenoma detection rate: meta-analysis of randomized controlled trials. Endoscopy 2018; 50: 846-860

[17] Triantafyllou K, Gkolfakis P, Tziatzios G et al. Effect of Endocuff use on colonoscopy outcomes: A systematic review and meta-analysis. World J Gastroenterol 2019; 25: 1158-1170

[18] Chin M, Karnes W, Jamal MM et al. Use of the Endocuff during routine colonoscopy examination improves adenoma detection: A meta-analysis. World J Gastroenterol 2016; 22: 9642-9649

[19] Jian HX, Feng BC, Zhang Y et al. EndoCuff-assisted colonoscopy could improve adenoma detection rate: A meta-analysis of randomized controlled trials. J Dig Dis 2019; 20: 578-588

[20] Rücker G, Schwarzer G. Resolve conflicting rankings of outcomes in network meta-analysis: Partial ordering of treatments. Res Synth Methods 2017; 8: 526-536

[21] Higgins JP, Thompson SG, Deeks J] et al. Measuring inconsistency in meta-analyses. BMJ 2003; 327: 557-560

[22] Dias S, Welton NJ, Caldwell DM et al. Checking consistency in mixed treatment comparison meta-analysis. Stat Med 2010; 29: 932-944

[23] Puhan MA, Schunemann HJ, Murad MH et al. A GRADE Working Group approach for rating the quality of treatment effect estimates from network meta-analysis. BMJ 2014; 349: g5630

[24] Higgins J, Altman D, Gotzsche P et al. The Cochrane Collaboration's tool for assessing risk of bias in randomised trials. BMJ 2011; 343: d5928-d5928

[25] Bhattacharyya R, Chedgy F, Kandiah K et al. Endocuff-assisted vs. standard colonoscopy in the fecal occult blood test-based UK Bowel Cancer Screening Programme (E-cap study): a randomized trial. Endoscopy 2017; 49: 1043-1050

[26] Biecker E, Floer M, Heinecke A et al. Novel endocuff-assisted colonoscopy significantly increases the polyp detection rate: a randomized controlled trial. J Clin Gastroenterol 2015; 49: 413-418
[27] De Palma GD, Giglio MC, Bruzzese D et al. Cap cuff-assisted colonoscopy versus standard colonoscopy for adenoma detection: a randomized back-to-back study. Gastrointest Endosc 2018; 87: 232-240

[28] Floer M, Biecker E, Fitzlaff $R$ et al. Higher adenoma detection rates with endocuff-assisted colonoscopy - a randomized controlled multicenter trial. PLoS One 2014; 9: e114267

[29] Jacob A, Schafer A, Yong J et al. Endocuff Vision-assisted colonoscopy: a randomized controlled trial. ANZ J Surg 2019; 89: E174-E178

[30] Rex DK, Slaven JE, Garcia J et al. Endocuff Vision reduces inspection time without decreasing lesion detection: a clinical randomized trial. Clin Gastroenterol Hepatol 2020; 18: 158-162.e1

[31] van Doorn SC, van der Vlugt M, Depla A et al. Adenoma detection with Endocuff colonoscopy versus conventional colonoscopy: a multicentre randomised controlled trial. Gut 2017; 66: 438-445

[32] Wada Y, Fukuda M, Ohtsuka K et al. Efficacy of Endocuff-assisted colonoscopy in the detection of colorectal polyps. Endosc Int Open 2018; 6: E425-E431

[33] von Figura G, Hasenöhrl M, Haller B et al. Endocuff vision-assisted vs. standard polyp resection in the colorectum (the EVASTA study): a prospective randomized study. Endoscopy 2020; 52: 45-51

[34] Rex DK, Repici A, Gross SA et al. High-definition colonoscopy versus Endocuff versus EndoRings versus full-spectrum endoscopy for adenoma detection at colonoscopy: a multicenter randomized trial. Gastrointest Endosc 2018; 88: 335-344.e2

[35] Karsenti D, Tharsis G, Perrot B et al. Adenoma detection by Endocuffassisted versus standard colonoscopy in routine practice: a clusterrandomised crossover trial. Gut 2020; 0: 1-6

[36] Kaminski MF, Wieszczy P, Rupinski M et al. Increased rate of adenoma detection associates with reduced risk of colorectal cancer and death. Gastroenterology 2017; 153: 98-105

[37] Aziz M, Desai M, Hassan S et al. Improving serrated adenoma detection rate in the colon by electronic chromoendoscopy and distal attachment: systematic review and meta-analysis. Gastrointest Endosc 2019; 90: 721-731.e1

[38] Aziz M, Fatima R, Lee-Smith W et al. Comparing endoscopic interventions to improve serrated adenoma detection rates during colonoscopy: a systematic review and network meta-analysis of randomized controlled trials. Eur J Gastroenterol Hepatol 2020: doi:10.1097| MEG.0000000000001844

[39] Anderson JC, Butterly LF, Goodrich M et al. Differences in detection rates of adenomas and serrated polyps in screening versus surveillance colonoscopies, based on the New Hampshire colonoscopy registry. Clin Gastroenterol Hepatol 2013; 11: 1308-1312 